Relations industrielles

Industrial Relations

\title{
Les étapes du syndicalisme agricole en France, Dr Louis Prugnaud, Ed. de l'Epi, Paris, 1963, 283 pages.
}

Jean Dosmond

Volume 19, numéro 1, janvier 1964

URI : https://id.erudit.org/iderudit/1021388ar

DOI : https://doi.org/10.7202/1021388ar

Aller au sommaire du numéro

Éditeur(s)

Département des relations industrielles de l’Université Laval

ISSN

0034-379X (imprimé)

1703-8138 (numérique)

Découvrir la revue

Citer ce compte rendu

Dosmond, J. (1964). Compte rendu de [Les étapes du syndicalisme agricole en France, Dr Louis Prugnaud, Ed. de l'Epi, Paris, 1963, 283 pages.] Relations industrielles / Industrial Relations, 19(1), 134-136.

https://doi.org/10.7202/1021388ar

Tous droits réservés (C Département des relations industrielles de l’Université Laval, 1964
Ce document est protégé par la loi sur le droit d'auteur. L'utilisation des services d'Érudit (y compris la reproduction) est assujettie à sa politique d'utilisation que vous pouvez consulter en ligne.

https://apropos.erudit.org/fr/usagers/politique-dutilisation/ 
faut pas oublier non plus que l'auteur se préoccupe constomment de l'application de la science économique à l'administration de I'entreprise.

Après une excellente préface du professeur Mourice Allais, M. Lesourne traite, assez longuement, dans son introduction, de la méthode scientifique et de la technique économique et puis du choix des critères économiques. Le volume comprend trois grandes parties.

La première réfère d̀ l'économétrie au service de l'entreprise. On y retrouve des chapitres sur les études commerciales, sur I'analyse théorique de la demande et sur les fluctuations de la conjoncture et deux derniers chapitres, I'un portant sur la discussion des données chiffrées et l'autre comprenant des exemples d'application de l'économétrie.

Le deuxième partie traite de la structure des coûts de l'entreprise. Deux courts chapitres: un premier sur la notion de coût: de la comptabilité à l'économique et un deuxième sur l'amortissement. Dans le troisième chapitre, Monsieur Lesourne fait une excellente analyse des coûts marginaux: théorie pure et applications concrètes.

Puis lo dernière partie développe les problèmes de synthèse: la régulation de la production et la gestion des stocks, la détermination des programmes optimums, les problèmes $d$ 'investissement et le calcul du revenu actualisé et enfin la politique de prix. Le seul chapitre réellement difficile est le deuxième de cette partie, chapitre qui présente des modèles mathématiques dont principalement le programme linéaire.

Dans une conclusion non moins excellente que les parties précédentes, l'auteur traite de la technique économique et de I'organisation de l'entreprise. II définit les missions des ingénieurs-économistes et parle du service économique dans l'entreprise.

\section{Bertrand Belzile}

Démocratie aujourd'hui. Ouvrage en collaboration par P. Antoine, F. Bloch-Lainé, F. Bourricaud, G. Burdeau, J.-Y. Calvez, A. Jeannière, G. Lavau, J.-L. Quermonne. Bibliothèque de la recherche sociale, Editions Spes, Paris, 1963, 188 pages.

《Autont la conviction démocratique est répandue aujourd'hui, autant elle manque le plus souvent de solide justification 》.
Cette affirmation que I'on trouve du début de l'introduction de cet ouvrage est pleinement vraie en ce qui concerne notre pays. Aussi nous croyons que les différents collaborateurs appartenant chacun d̀ des disciplines diverses ont rendu un grand service à tous ceux qui sont soucieux du fonctionnement efficace et de la survie de la démocratie.

On reprend ici, pour les rendre accessibles à un public plus vaste, une série d'articles publiés dans la revue de l'Action populaire en 1960-61-62.

L'ouvrage est divisée en deux parties: «Les raisons de la démocratie » et \& La démocratie en pratique ». La première partie comprend trois essais: «Du pouvoir personnel à la démocratie », «Démocratie et participation 》, «Liberté et pouvoir». La seconde en comprend cinq: «Destiin de l'idée démocratique 》, «Démocratiser la république », «La Polyarchie ou la société raisonnable», «Pouvoir économique et démocratique », \&Des démocraties qui fonctionnent $\gg$.

Souf un chapitre où il est particulièrement question de la situation française, tous les outres possèdent un caractère général.

Les auteurs ovaient pour préoccupation de réexaminer les raisons de la démocratie et d'apprécier le fonctionnement actuel des régimes démocratiques. L'ensemble de ces analyses, sans constituer un traité complet sur la question, est sûrement une contribution très valable et susceptible d'éclairer tous ceux qui veulent revivifier nos sociétés qui sont en train de pérécliter sous la menace du «consentement» et du «contentement 》.

\section{Gérard Dion}

\section{LES ETAPES DU SYNDICALISME AGRICOLE} EN FRANCE, Dr Louis Prugnaud, Ed. de I'Epi, Paris, 1963, 283 poges.

A tous ceux épris d'histoire rurale, Les Etapes du Syndicalisme agricole en France retracent comment celui-ci, profondément influencé par les partis politiques, a rendu à l'agriculture française d'importants services économiques. Aussi curieux que celo puisse paraître, il n'existe pas d'étude d'ensemble sur I'histoire syndicale agricole et l'auteur lui-même cite cette phrase extraite de I'ouvrage magistral de Marcel Bloch, Les Caractères originaux de I'histoire rurale 
française. «II est des moments où une synthèse fut elle prématurée rend plus de services que beoucoup de travaux d'analyse $\gg$. L'ouvrage est divisé en trois parties chionclugiques: La première étudie la période de 1884 à 1890; la seconde oborde l'ètude de la Corporation paysanne créée par le gouvernement du Maréchal Pétain sous l'occupation, le 2 décembre 1940; la troisième débute après la publication de I'ordonnance d'Alger du 26 juillet 1944 déclarant nulle la loi précédente et s'étend jusqu'ò novembre 1962.

Après un bref roppel des origines politiques, économiques, sociales et législatives du syndicalisme, l'auteur étudie le développement et le coractère des Syndicats et des Unions. Puis il passe en revue l'action économique et sociale des syndicats locaux et les interventions des unions dont l'action va s'amplifiant. Lo loi du 12 mors 1920 leur reconnait le droit d'exercer les droits de la partie civile. La Doctrine de I'Union Centrale des Syndicats agricoles s'élabore. Nous retrouvons la \& Droite» telle que la présente A. Siegfried (Tableau des Partis de Francel ovec ses deux tendances dont l'une s'inspire de Le Play et de Meuy. Ou côté économique il faut s'efforcer de remédier aux traités libre-échonge de Nopoléon III (loi douanière de 1892); sur le plan politique c'est la lutte à la fois contre les idées républicaines des Constituants de 1789 et contre le socialisme. En 1919 la Confédération Nationale des Associations Agricoles tient son premier Congrès à Paris. Elle en tint dix-huit. La crise de 1929, l'indiscipline des Associotions, les élections conservatrices de 1932 vont renforcer la Sté des Agriculteurs de France tandis que la C.N.A.A. tiendra son dernier Congrès en 1935. C'est la naissance des Associations Spécialisées mais aussi la résistance aux lois socioles et la lutte contre l'action du B.T.T. Le conservatisme de la rue d'Athènes, les controdictions syndicales, tels sont les traits essentiels du syndicalisme agricole français à la veille de 1940. Le vieillissement des structures économiques et sociales, le respect des principes de liberté institués par les législateurs de 1884, tels étaient aussi les vrais problèmes

Le ler juillet 1940, I'Armistice est conclu avec I'Allemogne, la 3ème République est remplacée por l'Etat français et c'est l'ovènement de la \& Révolution Notionale». La droite paysonne reprend les théries des Catholiques Sociaux: famille, religion, propriété, patronage sont les bases nécessaires du véritable régime social. La corporation paysanne (qui n'a rien à voir avec les cor- porations du Moyen Age) prend forme. A la base de l'organisation: le syndicat local corporatif: il n'y en ouro qu'un par circonscription. Au sommet le Conseil National Corporatif et la Chambre syndicale Nationale qui donnoit surtout des avis. . Tout un appareil administratif est mis en place... avec les Cadres de l'Union Notionale des Syndicats agricoles, dissoute. L'accueil des masses paysannes est sans enthousiasme, l'étatisation gagne du terroin ò l'échelon national et finalement la fusion du Ministère de l'Agriculture et du Ravitaillement discrédite les mesures du Gouvernement auprès des paysans.

C'est à Alger qu'est prise l'ordonnance du 26 juillet 1944 abolissant la Corporation. Après diverses tentotives de mouvements d'obédience communiste ou socialiste, le $\mathrm{Mi}$ nistère de I'Agriculture échoit à $\mathrm{Mr}^{\prime}$ Pfimlin (Maire de Strosbourg) représentant du Mouvement Républicain Populaire (M.R.P.) en 1946 et c'est le retour à la liberté syndicale. La C.Q.A. préparée en octobre 1944 après la libération du Territoire reçoit deux rôles: coordonner et représenter. Mais ce dernier attribut sera ò l'origine de la grave crise qui emportera la Confédération car la représentation est une tôche syndicale et la C.Q.A à la différence de la C.Q.T. n'est pas un syndicat.

Un excellent organigramme détaille le méconisme mis en place suivi de l'étude détaillée de la F.N.S.E.A.: Composition, effectifs, pourcentages, qu'est sa doctrine sinon le libéralisme économique «accompagné du vieil individualisme qui a régné au 19ème siècle 》

Peu à peu la F.N.S.E.A. sous I'influence du Cercle National des Jeunes Agriculteurs s'oriente vers une vue plus saine des choses. Cclui-ci voit son influence s'accroitie sur le plan national: Conseil Economique et Social, Commission Notionale de l'Agriculture, Conseil National de la Vulgorisation et du Progrès Agricole, Conseil d'administration de la Mutualité Sociale Agricole. Mais il reste tant à faire pour résoudre les problèmes actuels, donner à l'agriculture la place qui doit lui revenir au sein du Marché commun. C'est vers le « Regroupement \$ que s'oriente ou début de décembre 1962 la F.N.S.E.A. Telle est l'action syndicale depuis 1946. L'auteur ne se propose pas de dépeindre la situation économique et sociale de l'agriculture mais il indique combien son retard sur l'industrie a nécessité de mesures de soutien, de protection, de transformation. En 1960 l'Agriculture française 
n'était pas prête d̀ supporter la suppression de I'index recommandé par le célèbre économiste libéral J. Rueff. . . de là les mesures sociales... la lutte pour les prix, l'organisation des marchés, l'action en faveur de l'exploitation familiale puis la loi d'orientation agricole alors que ce livre allait poraître.

Sachons gré à l'auteur de donner oux agriculteurs cette excellente histoire du syndicalisme agricole. Ils y verront l'évolution de leurs associations et seront ainsi d̀ même d'apprécier l'efficacité de leur action, de constater leurs succès et leurs lacunes. Sous une forme maniable, elle constitue un tableau d'ensemble du monde agricole et rural, de ses luttes, de ses problèmes. Elle met en lumière le rôle octif que jouent toujours les paysans français dans la vie politique et économique d'un pays où plus d'un cinquième est rural malgré trois guerres et la désertion des campagnes. Elle souligne la nécessité d'un occroissement de la productivité qui seul permettra à la paysannerie d'augmenter sa part de revenu National. L'auteur ne dit presque rien des lois sur le métayage et le fermage de 1946 et ne foit pas ressortir combien la législation actuelle tend à «minimiser la vieille fortune acquise * ainsi que le remarque le $\mathrm{Dr}$ Savatier. Toute histoire peut paraître incomplète vue par un seul observateur, mais le mérite est grand de décrire des événements où tant de passions et d'intérêts se sont trouvés mêlés. II l'est d'autant plus que les ouvrages objectifs et scientifiques se limitent presque à l'oeuvre de Michel-AngeLaribe. L'ouvrage comporte d'assez nombreuses références en bas de page, des extraits de la presse, quelques cartes, une bibliogrophie et en annexe la loi du 21 mars 1884 qui levait les anciens obstacles juridiques. Les Etapes du Syndicalisme Agricole en France sont aussi la contribution de l'auteur au progrès et à l'amélioration des conditions de son pays et de so petite Commune natale limousine.

\section{Jean Dosmond}

Ethique sociale, tome 1: Les principes de la doctrine sociale. Par Arthur Utz, professeur à l'Université de Fribourg, Suisse. Editions Universitaires, Fribourg, Suisse. 1960, 419 poges.

L'intention de l'auteur est de présenter d'une manière systématique les normes fondomentales qui régissent la société comme telle. C'est seulement le premier volume d'une série qui comprendra ultérieurement la philosophie du droit, I'ordre social, I'ordre économique et l'ordre politique.

L'éthique sociale, telle que conçue par l'auteur et c'est de cette façon que l'on doit l'envisager est une discipline nettement distincte des outres sciences sociales, de l'éthique individuelle et de la théologie sociale. Après un chapitre introductif où l'auteur donne un aperçu historique du développement des sciences aux prises avec le social, il entre pleinement dans son sujet: Définition du social et de la société; les fondements de l'éthique sociale et son caractère scientifique; comment l'éthique so. ciale se distingue de la sociologie, de la philosophie sociale et de l'éthique individuelle; la nature sociale de I'homme; le bien commun; la réalisation du bien commun par la justice et l'amour; société et autorité; l'agir social; la question sociale; le personnalisme, expression de la doctrine sociale chrétienne; les diverses formes de la vie sociale; structure de l'éthique sociale. L'ouvrage comprend deux appendices et une bonne bibliographie internationale. Le premier oppendice reproduit les textes de $\mathrm{S}$ Thomas sur la nature sociale de l'homme, le second les textes du même auteur sur le bien commun.

Cet ouvrage est tout d̀ fait remarquable. L'auteur, bien au fait des données relevant des autres sciences sociales, sait utiliser ce qu'elles apportent tout en s'en tenant rigoureusement oux méthodes propres d̀ sa discipline. Sous ce rapport, en plus de so valeur intrinsèque exceptionnelle, il est un modèle du genre et se révèle un instrument extrêmement formateur pour les professeurs et les élèves qui s'en serviront. Les principes de la doctrine sociale d'Arthur Utz doit se trouver entre les moins de tous les professeurs en cette motière et porticulièrement chez ceux qui ont prétention d'être les disciples de S. Thomas. Nous espérons que l'auteur sera en mesure de publier le plus tôt possible les autres tomes qu'il annonce de son Ethique sociale.

\section{Gérard Dion}

EN GREVE: Ouvrage écrit en collaboration par Jean-Paul Lefebvre, Jean Francoeur, Pierre Vodeboncoeur et Jean-Louis Roux. Préface de Jean Marchand. Les Editions du Jour. Montréal, 1963. 280 poges.

Cet ouvroge retrace I'histoire de certaines grèves menées par la Confédération des syn- 